\title{
Editorial
}

\section{Epilepsy and Depression: A Bidirectional Relationship}

The study titled "High frequency of depressive symptoms among adults with epilepsy: Results from a hospital-based study" addresses an important but often neglected domain of clinical practice in epilepsy. ${ }^{[1]}$ The lifetime prevalence of depression in epilepsy has been reported to be as high as $55 \%$ by some authors. Depression worsens quality of life in patients with epilepsy and is a treatable disorder. Depression can lead to seizures by means of sleep deprivation, and seizures can lead to depression both through biological mechanisms and the psychosocial impact that epilepsy has. ${ }^{[2]}$ In fact, increase in the prevalence of suicide in patients with epilepsy and depression as compared to general population is a well-documented fact. ${ }^{[3]}$ Similarly, medication adherence is poor in epileptic patients who are depressed. Hence, screening, assessing, and managing comorbid depression in patients with epilepsy should be an integral component of epilepsy clinics. Epileptogenic properties of some antidepressants and fear of medicalizing the psychosocial impact of epilepsy as "depressive disorder" has held back effective identification and treatment of depressive disorder. Effective liaison between neurologists and psychiatrists is invaluable in this regard. There has been scant research on the bidirectional relationship between depression and epilepsy and effective treatment options for the same. In this background, the study tries to fill some vital gaps in the jigsaw.

The focus of the study has been on identification of frequency of depressive symptoms in epilepsy and the important mediating sociodemographic and clinical variables. However, the highlight of the study has been the emphasis on the use of a structured screening instrument for systematic identification of cases of epilepsy with comorbid depression. In this study, a validated regional language version of patient health questionnaire has been used. ${ }^{[4]}$ The other instruments recommended for this purpose include Primary Care Evaluation of Mental Disorders screening questionnaire for depressive symptoms (PRIME-MD) patient questionnaire, the Centre for Epidemiologic Studies Depression Scale, and Beck's and Hospital Anxiety and Depression scales. ${ }^{[5,6]}$

The study has identified the type of seizure and frequency of seizure episodes as important mediators in this bidirectional relationship. Lack of emphasis on psychological variables including previous and family history of mood disorders have been listed as potential limitations of the study by the authors. A combination of biological and psychological mechanisms is likely to contribute to the complex interplay between the two disorders.

Simply prompt identification of comorbidity would be insufficient without appropriate management strategies. Although clear guidelines are lacking, a variety of antidepressants with minimal or no epileptogenic property can be safely used. Psychological therapies including cognitive behavioral therapy have also been suggested as valuable strategies. Use of novel internet-based therapeutic applications has been emphasized in recent studies. ${ }^{[7]}$ Any of these treatment modalities as stand alone or in combination are likely to be effective in patients with epilepsy and comorbid depression. Early identification and treatment is likely to be invaluable.

A variety of relevant questions remain unanswered due to the paucity of research in the field. There is a need to identify personalized treatment strategies based on type, frequency, and severity of seizures as well as severity and nature of depressive symptoms rather than a "one size fits all" approach. On a more fundamental level, components of the biological and psychological interplay between the two disorders need to be discerned for a better understanding of this complex but interesting field of research. This is likely to have significant implications in day-to-day clinical practice as well as considering the high prevalence of these two common disorders. A multidisciplinary approach in research as well as clinical practice needs emphasis to answer the yet unanswered questions and for effective epilepsy care.

Sundar Gnanavel

Department of Child and Adolescent Mental Health Services, Northumberland Tyne and Wear NHS Foundation Trust, Morpeth, UK

Address for correspondence: Dr. Sundar Gnanavel, Northumberland Tyne and Wear NHS Foundation Trust, Morpeth, UK E-mail: sundar221103@yahoo.com

\section{REFERENCES}

1. Chandrasekharan SC, Menon V, Wadwekar V, Nair PP. High frequency of depressive symptoms among adults with epilepsy: Results from a hospital based study. J Neurosci Rural Pract 2017;8:S13-S9.

2. Lambert MV, Robertson MM. Depression in epilepsy: Etiology, phenomenology, and treatment. Epilepsia 1999;40 Suppl 10:S21-47.

3. Mendez MF, Doss RC. Ictal and psychiatric aspects of suicide in epileptic patients. Int J Psychiatry Med 1992;22:231-7.

4. Poongothai S, Pradeepa R, Ganesan A, Mohan V. Reliability 
and validity of a modified PHQ-9 item inventory (PHQ-12) as a screening instrument for assessing depression in Asian Indians (CURES-65). J Assoc Physicians India 2009;57:147-52.

5. Spitzer RL, Williams JB, Kroenke K, Linzer M, deGruy FV $3^{\text {rd }}$, Hahn SR, et al. Utility of a new procedure for diagnosing mental disorders in primary care. The PRIME-MD 1000 study. JAMA 1994;272:1749-56

6. Radloff LS. The CES-D scale: A self-report depression scale for research in the general population. Appl Psychol Meas 1977;1:385-401.

7. Meyer B, Weiss M, Holtkamp M, Arnold S, Brückner K, Schröder J, et al. Protocol for the ENCODE trial: Evaluating a novel online depression intervention for persons with epilepsy. BMC Psychiatry 2017;17:55.
This is an open access article distributed under the terms of the Creative Common Attribution-NonCommercial-ShareAlike 3.0 License, which allows others to remix, tweak, and build upon the work non-commercially, as long as the author is credited and the new creations are licensed under the identical terms.

\begin{tabular}{|l|l|}
\hline \multicolumn{2}{|c|}{ Access this article online } \\
\hline Quick Response Code: & Website: \\
\hline
\end{tabular}

How to cite this article: Gnanavel S. Epilepsy and depression: A bidirectional relationship. J Neurosci Rural Pract 2017;8:S5-6. 